\title{
Mapeando a formação do enfermeiro no Brasil: desafios para atuação em cenários complexos e globalizados
}

\author{
Mapping nursing training in Brazil: challenges for actions in \\ complex and globalized scenarios
}

Mirna Albuquerque Frota (http://orcid.org/0000-0003-3004-2554) ${ }^{1}$

Mônica Carvalho de Mesquita Werner Wermelinger (http://orcid.org/0000-0001-5861-5479) ${ }^{2}$

Luiza Jane Eyre de Souza Vieira (https://orcid.org/0000-0002-5220-027X) ${ }^{1}$

Francisco Rosemiro Guimarães Ximenes Neto (https://orcid.org/0000-0002-7905-9990) ${ }^{3}$

Raquel Santos Monte Queiroz (https://orcid.org/0000-0001-7788-7330) ${ }^{1}$

Rosendo Freitas de Amorim (https://orcid.org/0000-0003-1498-8999) ${ }^{1}$

${ }^{1}$ Programa de Pós-

Graduação em Saúde

Coletiva, Universidade de

Fortaleza. Av. Washington

Soares 1321, Edson Queiroz.

60811-905 Fortaleza CE

Brasil.mirnafrota@unifor.br

${ }^{2}$ Departamento de

Administração e

Planejamento em Saúde,

Escola Nacional de Saúde

Pública Sergio Arouca

Fiocruz. Rio de Janeiro RJ

Brasil.

${ }^{3}$ Centro de Ciências da

Saúde, Universidade

Estadual Vale do Acaraú.

Sobral CE Brasil.

\begin{abstract}
The article discusses the professional formation of nurses, implications of the increase in the number of Higher Education Institutions and their distribution in Brazil. It considers the results of the Nursing Profile Survey in Brazil, carried out with 35,916 nursing professionals, in 2013. The analysis that characterizes the trajectory of undergraduate nursing in this article is structured in three dimensions: the increase in the number of undergraduate and postgraduate nursing education institutions; the boom in nursing schools and the public vs. private relationship; and the territorial distribution of the registered nurse in Brazil. The increase in the number of Nursing Education Institutions implies an exponential formation, with a predominance of private schools in undergraduate and postgraduate courses. The courses seek to align themselves with changes in health and society, but it is crucial to equalize the territorial asymmetries between the undergraduate and graduate training institutions, the overconcentration and care gaps resulting from the insufficiency of nurses per inhabitant, as well as to qualify the nurses for the exercise of their professional activities in the face of global changes.

Key words Nurse, Education Nursing Diploma Programs, Education Nursing Graduate, Professional Training, Professional Practice
\end{abstract}

Resumo O artigo discorre sobre a formação profissional do enfermeiro, implicações da expansão das Instituições de Ensino Superior e a distribuição dessas no Brasil. Considera os resultados da Pesquisa Perfil da Enfermagem no Brasil, realizada com 35.916 profissionais de Enfermagem, em 2013. A análise que caracteriza a trajetória da graduação em enfermagem neste artigo, estrutura-se em três dimensões: a expansão da formação do enfermeiro na graduação e pós-graduação; o boom de escolas de enfermagem e a relação público $x$ privado; e, a distribuição territorial do profissional enfermeiro no Brasil. O crescimento de Instituições de Ensino em Enfermagem implica em uma formação exponencial, com predominio de escolas privadas na graduação e na pós-graduação; os cursos buscam alinhar-se às mudanças na saúde e sociedade, mas urge equalizar as assimetrias territoriais entre as instituições formadoras na graduação e pós-graduação, a superconcentração e vazios assistenciais decorrentes da insuficiência de enfermeiros por habitantes, bem como qualificar o enfermeiro para o exercício profissional ante as transformações globais.

Palavras-chave Enfermeiro, Programas de Graduação em Enfermagem, Educação de Pós-Graduação em Enfermagem, Capacitação Profissional, Prática Profissional 


\section{Introdução}

A formação profissional atualiza-se na medida em que inscreve-se nos cenários educativos, laborais, políticos, econômicos e sociais que seguem as transformações globais ${ }^{1}$. Estes convivem com transições demográficas, revoluções tecnológicas, educacionais, culturais e seus arcabouços epistemológicos, que alicerçam ou impulsionam as instituições à transformação, adaptação e inovação em um mundo conectado².

Este entendimento é recursivo quanto aos desafios que pautam a formação acadêmica para situá-la nesses contextos transformadores ${ }^{3}$. A formação plural prediz impactos nos indicadores de saúde, melhoria da qualidade de vida das populações assegurando o cunho social da prática profissional do enfermeiro ${ }^{4}$. Nesse contexto, o artigo versa sobre a formação profissional do enfermeiro, em nível de graduação e pós-graduação e suas implicações, considerando os resultados da Pesquisa Perfil da Enfermagem no Brasil $(\mathrm{PPEB})^{5,6}$.

Deste modo, a análise dos resultados que caracterizam a trajetória da graduação em enfermagem, estrutura-se em três dimensões distintas, porém interconectadas: (i) a expansão da formação do enfermeiro na graduação e pós-graduação; (ii) o boom de escolas de enfermagem e a relação público x privado; e, (iii) a distribuição territorial do profissional enfermeiro no Brasil, considerando as duas anteriores.

Justificam-se estudos nesta linha analítica no sentido de que a formação profissional do enfermeiro necessita desenvolver uma visão globalizada e inserir-se no mundo competitivo que, cada vez mais, produz e difunde o conhecimento. Por outro lado, urge que essa realidade seja modificada e que novas propostas materializemse, transformando o perfil de formação do enfermeiro. No entanto, parecem coexistir nichos geopolíticos em que o profissional enfermeiro se adapta com facilidade, mesmo com o atual perfil de formação, com outros, em que ele tem suas competências, habilidades e atitudes confrontadas ao deparar-se com diferentes demandas mercadológicas.

Inserido nessa discussão e defendendo a existência de cenários formadores compactuados com as transformações do Século $\mathrm{XXI}^{1-3}$, $\mathrm{o}$ artigo analisa nexos que perpassam o crescimento acelerado da formação do enfermeiro e a ocorrência da privatização do ensino superior de Enfermagem, bem como a heterogênea intensidade e distribuição territorial desses aspectos no Brasil, levando à convivência entre cenários de atendimento nos moldes preconizados por organismos internacionais, com outros de carência absoluta.

\section{Crescimento acelerado da formação do enfermeiro na graduação e pós-graduação}

No Brasil, a formação do profissional enfermeiro busca alinhar-se com as Diretrizes Curriculares Nacionais (DCN) no sentido de transpor o paradigma Flexneriano que modelou as matrizes curriculares dos cursos da saúde ${ }^{7}$. Reconhecese que os Projetos Pedagógicos dos Cursos (PPC) de Enfermagem buscam transpor matrizes curriculares centradas em temas que priorizam aspectos biológicos e práticas medicalizantes. Dessa forma, os projetos intentam oferecer à sociedade a formação de um profissional com competências, habilidades e atitudes éticas que respondam às demandas que aportam os serviços de saúde $\mathrm{e}$ a intersetorialidade ${ }^{8}$.

Nesse sentido, as $\mathrm{DCN}^{9}$ norteiam a elaboração dos currículos para os cursos de graduação seguidos pelas Instituições de Ensino Superior (IES), garantindo, entre outros propósitos, a flexibilidade acadêmica a fim de assegurar uma formação básica sólida, capaz de preparar o profissional para lidar com os desafios e as rápidas transformações globais.

Em contraponto, mostra-se recorrente na literatura os debates e embates sobre a materialidade das DCN nos currículos da área da saúde e sua repercussão na formação do profissional ${ }^{10}$. Ainda não se visualizam concretas articulações teórica e prática, posturas profissionais com contornos de uma prática social que responda às necessidades de saúde da população, bem como o perfil profissional esperado para dar conta das demandas assistenciais do Sistema Único de Saúde (SUS) ${ }^{7-8,11}$.

Porém, reconhece-se nas últimas décadas que os avanços na formação do enfermeiro são frutos de mudanças paradigmáticas, como tentativa de superar o pensamento simplificado e fragmentado da realidade, para construir uma visão integrada, complexa, polissêmica, multifacetada e inovadora. Em sintonia com as políticas de educação e saúde identificam-se nos currículos incentivos para que o estudante torne-se sujeito central de sua formação, capaz de transitar na flexibilidade das matrizes curriculares e agir ante as mudanças e incorporações de conhecimento ${ }^{11,12}$.

Há um consenso entre estudiosos da área sobre a recorrência de debates pautados na formação de um profissional generalista, ciente das 
singularidades sociossanitárias, da importância dos avanços tecnológicos, das incertezas políticas e econômicas, além de outros desafios, o que demandam as IES repensarem os modelos de formação profissional no Século XXI ${ }^{1-4,6}$.

Alinhada com essas transformações, a Universidade do Chile desenvolve um modelo diferenciado na formação profissional dos estudantes de Enfermagem, embasado na crescente migração entre os países sul americanos. Esse modelo agregou inovações curriculares que respondessem a essas adaptações culturais, a exemplo das questões raciais, o respeito à diversidade e introdução de práticas não discriminatórias e xenofóbicas. A experiência tem sido satisfatória, mas em constante atualização para atingir o objetivo esperado. $\mathrm{Na}$ análise dessa experiência, pondera ser indispensável que os profissionais de enfermagem contribuam no avanço de uma atenção à saúde equitativa, inclusiva e com coerência cultural para a construção de uma identidade profissional ${ }^{12}$.

Além do posicionamento e compreensão da diversidade transcultural é mister que os estudantes desenvolvam o pensamento crítico durante a formação profissional, com possibilidades de trilhar na vertente da pesquisa e atividades de extensão como mecanismo de aprimoramento dessa visão crítica ${ }^{13}$.

Nessa sintonia, Linda Fergunson ${ }^{14}$, especialista em educação e formação em enfermagem (Associação de Enfermeiros Canadenses), destaca que a evolução das pesquisas em Enfermagem reflete sobre a ausência de uma experiência clínica qualificada. Acrescenta que prosseguir com a ideia de os estudantes continuarem fazendo as mesmas coisas e de modo repetido, pode certificar os créditos a obtenção do diploma. Porém, considerar uma formação integral e qualificada requer mais que a experiência clínica repetitiva.

É neste implexo de saberes que a formação acadêmica dos cursos de enfermagem deve pautar-se, indo além da "participação técnico-científica”, deve privilegiar, sobremodo, temas sociais, políticos, econômicos, éticos e legais capazes de impulsionarem um agir responsável perante a sociedade. Diversos autores apontam como necessidade imperiosa que a educação resgate e ressignifique uma formação voltada a cidadania, com uma liderança responsável e reconhecida por uma prática ética que ressalte os valores morais da profissão ${ }^{15}$.

$\mathrm{Na}$ articulação de resgate da convivência ética e colaborativa, o Marco para Ação em Educação Interprofissional e Prática Colaborativa ${ }^{16}$, alerta e convoca as instituições formadoras de profissionais para o setor saúde sobre a exigência do trabalho em rede, cooperativo e articulado, troca de saberes entre as profissões com apreensão interdisciplinar.

No Brasil, a Constituição Federal ${ }^{17}$ ancorada nos princípios epistêmicos e organizativos do SUS, define a saúde como bem social, um direito do cidadão e dever do Estado. Impulsionados pela reorientação do modelo de atenção à saúde e exigência da atenção contemplando os níveis de complexidade, e sob influência da Lei de Diretrizes e Bases da Educação (LDB) ocorre a expansão do ensino superior, com o predomínio do setor privado, incialmente, com o intento de suprir os vazios assistenciais decorrentes da escassez de recursos humanos na área da saúde.

Com a criação e implantação do Programa de Saúde da Família (PSF), posteriormente Estratégia Saúde da Família (ESF), o profissional enfermeiro, que compõe a equipe mínima, firma-se como protagonista na ampliação da cobertura e garantia do acesso a Atenção Primária à Saúde (APS) à população, demandado pelo incremento da descentralização de ações e serviços e o fortalecimento da gestão sanitária nos municípios brasileiros.

Após o ano 2000, com o avanço na implantação e implementação de políticas sociais e sanitárias inclusivas, ao mesmo tempo há proliferação de cursos universitários na área da saúde. Entretanto, pondera-se sobre a sustentação de um ensino de qualidade, plural e conectado com a empregabilidade em espaços urbanos e rurais, na perspectiva de multipaíses, ainda que cada área geográfica seja detentora de problemas locais, diversidade cultural e um perfil epidemiológico local, mas inscritos e relacionados com desafios globais.

\section{O boom de escolas de enfermagem e a relação público $x$ privado}

O número de escolas de enfermagem no Brasil e consequente aumento do número de profissionais formados por ano e disponibilizados para um mercado de trabalho com múltiplas necessidades de saúde e demandas sociais, exige uma reorientação de rumos nesta seara educacional ${ }^{6}$.

No final da década de 1990, o ingresso no curso superior no Brasil estava associado à posição estrutural do indivíduo. Com a crescente oferta de faculdades privadas, essa associação deixa de ser linear e inicia-se uma ascensão exponencial ${ }^{18}$. Além da expansão da oferta de faculdades parti- 
culares, cabe sinalizar a criação de programas de bolsas de estudo para o ensino superior (público e privado), contribuindo para que uma parcela da população - incluindo indivíduos pertencentes às camadas mais pobres da sociedade - tenha acesso a esse nível de ensino ${ }^{19}$.

A obtenção de um diploma foi se tornando requisito para o emprego. As faculdades privadas, movidas por um empresariado da educação, ampliaram a oferta de vagas para setores menos favorecidos da população. A expansão do ensino superior tem se apoiado no fenômeno da certificação, pois o mercado de trabalho eleva a exigência de diplomação para postos antes ocupados por profissionais de nível médio ${ }^{20}$. Esse processo induz que trabalhadores inseridos no mercado de trabalho procurem formação de nível superior, ao perceberem ameaça com a oferta de profissionais de maior titulação, no entanto, dispostos a exercerem os mesmos cargos e funções ${ }^{21}$.

A expansão da escolaridade verificada entre o período de 2002 e 2012 é expressão de múltiplas determinações, como mudanças no mundo do trabalho que exige readaptação dos profissionais ao mercado. Os números disponibilizados pelo Instituto Nacional de Estudos e Pesquisas Educacionais Anísio Teixeira Legislação (INEP) para o ensino de nível Superior, como um todo, têm inquestionável relevância, ao atestar que, se no ano de 2000 tinha-se 352.305 concluintes do ensino superior no Brasil, em 2012 esta cifra atinge 1.050 .413 registrando aumento de $198 \% \%^{22,23}$.

O setor saúde traz o diferencial de não substituir, em sua totalidade, o elemento humano pela tecnologia, apesar de ser um setor de intensa e acelerada incorporação tecnológica, requerendo, cada vez mais, trabalhadores credenciados a atuar nesse mercado em constante mudança. No setor saúde prevalecem as tecnologias leves e a subjetividade do trabalho humano. É neste setor que a expansão das IES encontrou espaço, reflexo e motivação exacerbados. Os cursos da área da saúde tiveram, no mesmo período analisado, incremento de $218 \%$ no número de concluintes, variando de 69.323 em 2000, para 215.074 em $2012^{24}$.

A Enfermagem com quantitativo hegemônico na área da saúde reflete essa realidade. Com a criação de 684 novos cursos (2000-2012) passou de 183 para 867 o que representava em 2012, 22\% dos concluintes da área da saúde. Alcançou, nesse período, um incremento de $450 \%$ de vagas ofertadas e de $750 \%$ do número de concluintes.

Até o final da década de 1960 tinha-se no Brasil 34 escolas de Enfermagem. Em 1988 com a proposta de universalização do direito à saúde, contabilizávamos, em todo o país, 103 cursos de Enfermagem $^{25}$. Esse número permaneceu com crescimento linear e modesto até 1995 quando o país contabilizava 108 cursos $^{26}$. No entanto, como observa-se no Gráfico 1, segue crescendo de forma acelerada, principalmente no setor privado ${ }^{27}$.

A questão da privatização da graduação em Enfermagem é recente, progressiva e, aparentemente, encontra-se em franca expansão. Se, entre aqueles enfermeiros formados na década de 1990, 35,1\% se graduaram em IES privadas; entre os que concluíram o curso na década de 2000, esse percentual chegou a $63,6 \%$ e atingiu $75,7 \%$ entre os que se formaram de 2010 a 2013. Assim, enfermeiros graduados até o final do Século XX eram, sobretudo, formados pelo poder público, correspondendo a 59,1\% do total de formados entre 1990 e 1999 . Já entre 2010 e 2013 apenas 19,0\% dos enfermeiros concluíram a graduação no setor público (Gráfico 1).

Em contraponto, enfrenta-se carência de enfermeiros no âmbito global. Estudo de coorte em realização na Tailândia com enfermeiros licenciados mostra a falta desse profissional, em nível preocupante. Identificou alta taxa de enfermeiros com intenção de abandonar a profissão e baixo número de ingressantes nos cursos ${ }^{28}$.

Soma-se ao imbróglio das implicações do elevado número de IES, questões sobre a utilização dos Cursos Técnicos em Saúde como: (1) "trampolim" de conteúdos e formação para a seleção ao nível superior de educação, sem nenhum compromisso com a atuação na área da saúde; (2) itinerário formativo dentro de uma carreira na saúde; ou (3) condição para a empregabilidade financiadora do ensino superior privado. $\mathrm{O}$ maior expoente na realização desses cursos encontra-se na equipe de enfermagem e na inciativa privada.

O grande número de técnicos e/ou auxiliares de enfermagem que estão se graduando, em algumas situações podem originar tensionamentos. Esses conflitos podem existir no decorrer da formação acadêmica, no exercício da prática profissional, no convívio com equipe de saúde e de enfermagem.

Os dados da $\mathrm{PPEB}^{5}$ demonstraram que mais de 31\% dos enfermeiros do Brasil têm o diploma de Técnicos ou Auxiliares de Enfermagem, tendo feito o curso antes da graduação; e, mais de um quarto dos enfermeiros exerce ou exerceu a função de nível médio ou fundamental. Outra informação desvelada pelos dados da pesquisa é que esse aspecto da formação do enfermeiro é mais 
comum entre os homens, dentre os quais $41,1 \%$ possuem diploma e $37,6 \%$ exerceram a função de auxiliar ou de técnico em enfermagem (Quadro 1).

Possivelmente a profissionalização técnica em enfermagem anterior à graduação possa sinalizar o imperativo desse profissional adentrar ao mercado de trabalho no setor saúde, considerado um importante complexo empregador. Por outro lado, no decorrer das dinâmicas de trabalho o técnico ou auxiliar identifica as urgentes demandas para ampliar a qualificação e tornar-se um profissional competitivo no cenário empregador. Ademais, há o desejo de ascender socialmente mediante a conclusão do ensino superior.

Ao situar essa compreensão, estudo que discorre sobre o processo de socialização e a transformação de técnicos de enfermagem em enfermeiros ${ }^{29}$, observou que essa socialização potencializa o entendimento das motivações decorrentes do desejo de ascensão profissional na enfermagem, que envolvem causas objetivas, como o anseio por melhor remuneração, e motivações subjetivas, como a aspiração por reconhecimento social.

Mesmo considerando o aumento exponencial de concluintes dos cursos de enfermagem entre as IES brasileiras, esse não foi suficiente - $\mathrm{e}$ nem planejado - para minimizar as assimetrias regionais na proporção de enfermeiro por número de habitantes. O que perdura é a concentração em centros urbanos em detrimento das áreas rurais, e entre as regiões mais desenvolvidas e populosas quando analisadas as demarcadas pelas seculares desigualdades sociais. Este perfil acompanha a distribuição desigual das IES que ofertam cursos de enfermagem de graduação e pós-graduação no país.

\section{Distribuição territorial do profissional enfermeiro}

Este cenário torna-se crítico nas Américas, África Subsaariana e Sudeste Asiático - continen-

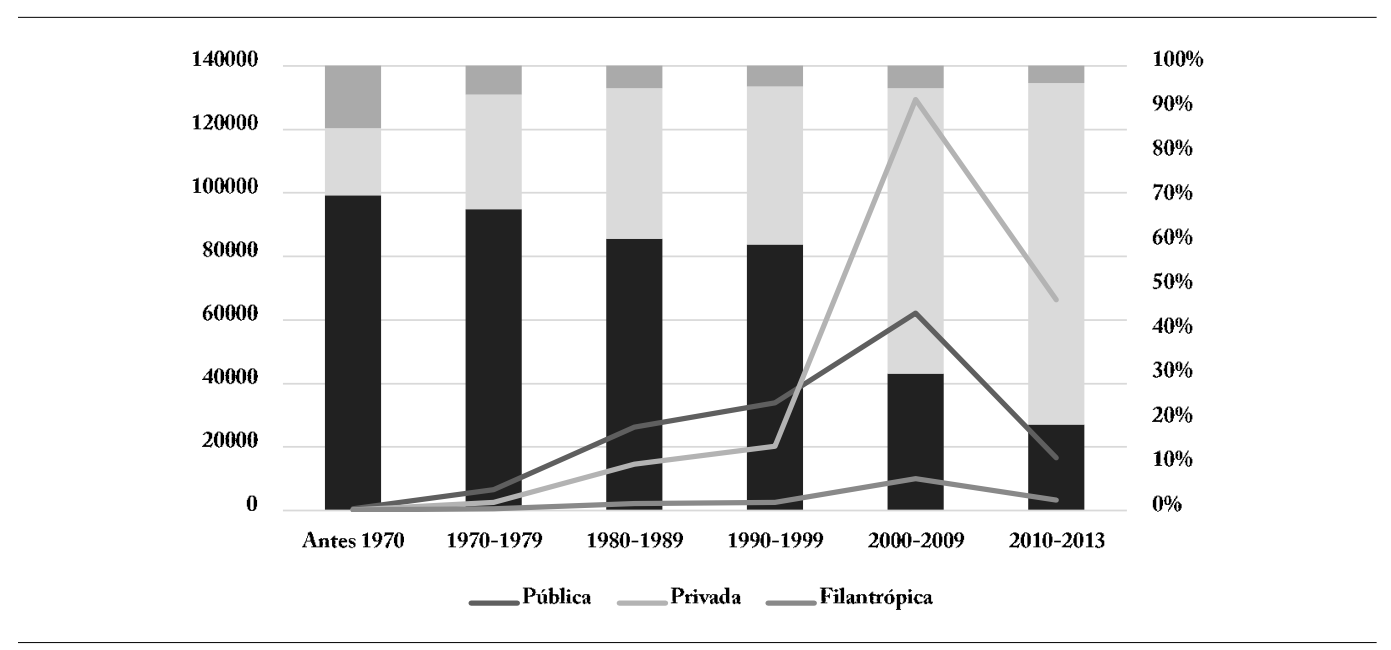

Gráfico 1. Enfermeiros por período de formação e tipo de IES.

Fonte: Pesquisa Perfil da Enfermagem no Brasil, Fiocruz, 2013.

Quadro 1. Enfermeiros que fizeram o Curso de Auxiliar ou Técnico de Enfermagem e exerceram a função. PPEB, 2013.

\begin{tabular}{|l|c|c|c|c|c|c|}
\hline \multirow{2}{*}{ Sexo } & \multicolumn{2}{c|}{$\begin{array}{c}\text { Fez Curso } \\
\text { Auxiliar ou Técnico }\end{array}$} & \multicolumn{2}{c|}{$\begin{array}{c}\text { Exerceu função } \\
\text { Auxiliar ou Técnico }\end{array}$} & \multicolumn{2}{c|}{ Total de Enfermeiros } \\
\cline { 2 - 7 } & $\mathrm{N}$ & $\%$ & $\mathrm{n}$ & $\%$ & $\mathrm{~N}$ & $\%$ \\
\hline Feminino & 106.868 & 29,9 & 90.806 & 25,4 & 357.552 & 100,0 \\
\hline Masculino & 22.789 & 41,1 & 20.854 & 37,6 & 55.401 & 100,0 \\
\hline Total & 129.657 & 31,4 & 111.660 & 27,0 & 412.953 & 100,0 \\
\hline
\end{tabular}

Fonte: Pesquisa Perfil da Enfermagem no Brasil, Fiocruz, 2013. 
tes que convivem com extrema pobreza, guerras, violências, conflitos políticos, fluxos migratórios, catástrofes ambientais e turbulências que requisitam intervenções do campo da saúde e carecem de profissionais com conhecimentos técnico, científico e culturalmente competentes ${ }^{30,31}$.

A Estratégia Global da Organização Mundial da Saúde (OMS) sobre Recursos Humanos para a Saúde: Força de Trabalho - 2030, define a agenda política para garantir uma força de trabalho que seja adequada ao propósito de atingir as metas dos Objetivos de Desenvolvimento Sustentável. Este documento de base analisa as implicações quantitativas e os requisitos para a sua implementação, preconizando como ideal, o índice de 4,45 profissionais entre médicos, enfermeiras e parteiras por 1.000 habitantes $^{32}$.

Esse documento, intitulado Health workforce requirements for universal health coverage and sustainable development goals, estima que para atingir o índice preconizado, só nas Américas, em 2013, o déficit de médicos era de cerca de 47.500 profissionais $(2,3 \%)$ e o de enfermeiras e/ou parteiras, acima de 516.000 profissionais (11,0\%).

No entanto, a Organização para a Cooperação e Desenvolvimento Econômico (OCDE) no documento Health at a Glance 2017: OECD Indicators $^{1}$, assinala a necessidade de 9,0 enfermeiros por mil habitantes para possibilitar acesso e atenção qualificada às populações.

Estudos atestam a existência de gaps assistenciais que confrontam a formação desse profissional. Ao mesmo tempo que há um quantitativo deslocado do mercado de trabalho, identificamse gaps pelo reduzido número de profissionais qualificados. Este contraditório tem se constituído desafio aos sistemas e serviços de saúde no cenário internacional e nacional. Pode-se entender que o fenômeno é multidimensional e considera vários aspectos, como oportunidades por melhores condições de trabalho, desenvolvimento profissional, qualidade de vida pessoal e familiar ${ }^{33}$.

No Brasil, este cenário é identificado quanto a concentração e assimetria na distribuição dos profissionais enfermeiros pelos Estados brasileiros e, mesmo, dentro deles. No Quadro 2, a distribuição desses profissionais não acompanha, proporcionalmente, a área territorial de cada Estado, nem mesmo a distribuição relativa da população entre eles.

Outro aspecto demonstrado (Quadro 2), é a superconcentração de enfermeiros nas capitais dos Estados. Nesse sentido, vale registrar que a maior concentração de enfermeiros numa capital foi observada em Aracaju, onde estão mais de $90 \%$ dos enfermeiros de Sergipe. Verifica-se que, com exceção do Distrito Federal, a taxa de enfermeiros por mil habitantes é, pelo menos $29 \%$ maior nas capitais do que no referido Estado - como no Rio de Janeiro - e pode chegar a ser $352 \%$ maior - como observado no cálculo das taxas de enfermeiros/1.000 habitantes em Florianópolis e em Santa Catarina.

O Distrito Federal $(4,07)$ e os Estados do Rio de Janeiro (2,75), Tocantins $(2,59)$ e Paraíba $(2,36)$, além das cidades de Belo Horizonte $(9,26)$, João Pessoa $(8,22)$, Porto Alegre $(7,97)$ e Vitória $(7,86)$ - respectivamente, capitais de Minas Gerais, Paraíba, Rio Grande do Sul e Espírito Santo - possuem as maiores taxas de enfermeiros/1.000 habitantes. Por outro lado, os Estados e capitais brasileiros com menores taxas desses profissionais/1.000 habitantes são o Pará $(1,11)$, Roraima (1,20), Alagoas $(1,24)$, Sergipe $(1,47)$, além das cidades de Boa Vista $(\mathrm{RR} / 1,67)$, Brasília $(\mathrm{DF} / 2,03)$, Porto Velho $(\mathrm{RO} / 2,25)$ e Macapá $(\mathrm{AP} / 2,70)^{34}$.

Outro aspecto desvelado pela PPEB é que as desigualdades intraestaduais podem ser maiores do que as interestaduais ou interregionais. Assim, o Estado de Minas Gerais, por exemplo, com a taxa de 2,06 enfermeiros por 1.000 habitantes está distante de atingir o índice mínimo ideal, preconizado pela OMS para profissionais de saúde - médicos, enfermeiras e parteiras (considerando que o contingente de enfermeiros e enfermeiros obstetras, na realidade brasileira, é sempre maior do que o contingente de médicos) - que é de 4,45 para cada 1.000 habitantes, até 2030. Já a capital de Minas, a cidade de Belo Horizonte, possui uma realidade bem diferente, com 9,26 enfermeiros/ 1.000 habitantes, valor que leva a cidade a superar até mesmo o recomendado pela $\mathrm{OCDE}^{1}$ para a categoria de enfermeiros, que corresponde a 9,0 enfermeiros/1.000 habitantes.

Diferente do que poderíamos imaginar, dois Estados da região Sul mostram uma desigualdade entre Capital x Interior também bem pronunciada. O Rio Grande do Sul tem uma taxa de 1,85 enfermeiros/1.000 habitantes enquanto sua capital Porto Alegre existem 7,97 enfermeiros/1.000 habitantes; semelhante ocorre no estado de Santa Catarina, 1,74 enfermeiros/1.000 habitantes, enquanto o índice de Florianópolis, a capital é de 7,84 enfermeiros/1.000 habitantes.

Sobre a atuação de enfermeiros no interior e em comunidades remotas é importante considerar que as populações que habitam nos territórios rurais possuem crenças que diferem das cultivadas em zonas urbanas quanto ao sentido 
Quadro 2. Características de distribuição e formação do enfermeiro no território brasileiro.

\begin{tabular}{|c|c|c|c|c|c|c|}
\hline Estados & $\begin{array}{l}\text { População } \\
\text { em } 2013 \\
\text { (n) }\end{array}$ & $\begin{array}{c}\text { População } \\
\text { na Capital } \\
(\%)\end{array}$ & $\begin{array}{c}\text { Enfermeiros } \\
\text { (n) }\end{array}$ & $\begin{array}{c}\text { Enfermeiros } \\
\text { na capital } \\
(\%)\end{array}$ & $\begin{array}{l}\text { Cursos de Pós- } \\
\text { Graduação } \\
\text { realizados por } \\
\text { enfermeiros }\end{array}$ & $\begin{array}{c}\text { Enfermeiros } \\
\text { graduados em } \\
\text { IES Privadas } \\
(\%)\end{array}$ \\
\hline \multicolumn{7}{|c|}{ Norte } \\
\hline Acre & 776.463 & 46,0 & 1.709 & 79,9 & 1.541 & 20 \\
\hline Amazonas & 3.807 .921 & 52,1 & 8.502 & 83,2 & 7.727 & 53,3 \\
\hline Amapá & 734.996 & 59,5 & 1.350 & 87,6 & 1.022 & 24,9 \\
\hline Pará & 7.969 .654 & 17,9 & 8.828 & 69,8 & 9.176 & 25,4 \\
\hline Rondônia & 1.728 .214 & 28,1 & 2.850 & 38,3 & 2.355 & 69,4 \\
\hline Roraima & 488.072 & 63,3 & 584 & 88,2 & 567 & 46,7 \\
\hline Tocantins & 1.478 .164 & 17,5 & 3.822 & 41,7 & 3.015 & 69,9 \\
\hline \multicolumn{7}{|c|}{ Nordeste } \\
\hline Alagoas & 3.300 .935 & 30,2 & 4.078 & 72,0 & 4.143 & 41,4 \\
\hline Bahia & 15.044 .137 & 19,2 & 27.489 & 65,7 & 28.303 & 52,2 \\
\hline Ceará & 8.778 .576 & 29,1 & 14.308 & 73,8 & 14.728 & 47 \\
\hline Maranhão & 6.794 .301 & 15,5 & 10.685 & 62,1 & 10.446 & 55,4 \\
\hline Paraiba & 3.914 .421 & 19,7 & 9.232 & 68,5 & 8.786 & 55,4 \\
\hline Pernambuco & 9.208 .550 & 17,4 & 16.624 & 65,3 & 17.508 & 42 \\
\hline Piauí & 3.184 .166 & 26,3 & 6.847 & 66,9 & 6.794 & 36,2 \\
\hline Rio Grande do Norte & 3.373 .959 & 25,3 & 6.490 & 61,7 & 6.527 & 29,5 \\
\hline Sergipe & 2.195 .662 & 28,0 & 3.228 & 90,2 & 2.451 & 38,4 \\
\hline \multicolumn{7}{|c|}{ Sudeste } \\
\hline Espírito Santo & 3.839 .366 & 9,1 & 9.607 & 28,5 & 7.980 & 68 \\
\hline Minas Gerais & 20.593 .356 & 12 & 42.498 & 54,0 & 35.636 & 65,5 \\
\hline Rio de Janeiro & 16.369 .179 & 39,3 & 44.977 & 50,5 & 52.898 & 54,5 \\
\hline São Paulo & 43.663 .669 & 27,1 & 105.438 & 60,2 & 106.352 & 68 \\
\hline \multicolumn{7}{|c|}{ Sul } \\
\hline Paraná & 10.997 .465 & 16,8 & 19.224 & 39,5 & 19.770 & 55,3 \\
\hline Rio Grande do Sul & 11.164 .043 & 13,2 & 20.629 & 56,7 & 22.271 & 52,1 \\
\hline Santa Catarina & 6.634 .254 & 6,8 & 11.523 & 30,9 & 12.889 & 56,5 \\
\hline \multicolumn{7}{|c|}{ Centro-Oeste } \\
\hline Goiás & 6.434 .048 & 21,7 & 11.801 & 60,5 & 10.421 & 70 \\
\hline Mato Grosso do Sul & 2.587 .269 & 32,2 & 4.627 & 50,9 & 4.088 & 55 \\
\hline Mato Grosso & 3.182 .113 & 17,9 & 6.408 & 62,6 & 6.119 & 53,2 \\
\hline Distrito Federal & 2.789 .761 & 100 & 11.354 & 49,9 & 9.507 & 57 \\
\hline
\end{tabular}

Fontes: Elaboração própria. IBGE e Pesquisa Perfil da Enfermagem no Brasil.

atribuído às doenças, o modo de percebê-las e as terapêuticas adotadas. Nesse cenário é fundamental compreender que muitas dessas situações não podem ser cuidadas com práticas lineares e protocolares, desenvolvidas para perfis epidemiológicos de áreas urbanas. As populações das zonas rurais exigem atributos diferenciados, destacando-se a competência cultural para efetivar a atenção qualificada ${ }^{35}$. Esse desafio tem impulsionado a organização da prática de enfermagem em países como Canadá, Estados Unidos da América e Austrália a realizarem mudanças na legislação e formação profissional, ampliando o escopo de atuação do enfermeiro ${ }^{36}$.

A tendência da concentração das IES na região Sudeste associa-se ao fato de ser essa a região que possui a maior produto interno bruto, grande parte da população brasileira, ter vivenciado intenso processo de industrialização anterior às demais regiões, além de ter um importante arse- 
nal tecnológico e o maior mercado de trabalho para todas as profissões, em especial as de formação universitária.

No Brasil, não existe uma formação nas graduações de Enfermagem que contemplem a realidade das áreas rurais. As bases curriculares são generalistas e não ponderam, de modo amplo, as especificidades geográficas. Até mesmo a oferta da prática às comunidades rurais ocorre somente quando as unidades formadoras julgam necessário ou há interesse por parte do discente ${ }^{37}$.

$O$ Quadro 2 registra o percentual de enfermeiros formados em IES privadas em todo o país, bem como uma taxa média de cursos de Pós-Graduação (formação profissional na modalidade de Residência/Programa de Residência, Especialização, Mestrado Profissional, Mestrado Acadêmico, Doutorado e Pós-Doutorado) realizados pelo quantitativo total de enfermeiros do Estado.

Observa-se peculiaridades na região Norte: tem-se os quatro Estados (Roraima, Amapá, Acre e Rondônia) com menor número absoluto de enfermeiros; estão entre os cinco menos populosos (Roraima, Amapá, Acre, Tocantins e Rondônia); Amapá e Roraima apresentam maior concentração populacional e de contingente de enfermeiros nas capitais. Por outro lado, o Acre, Amapá e Pará predominam a formação do enfermeiro em IES públicas; e, no Amapá e Tocantins estão as menores oportunidades para cursar uma PósGraduação.

$\mathrm{Na}$ região Nordeste identificam-se as situações: Alagoas, Sergipe, Maranhão e Ceará registram menores números de enfermeiros; um deles é o menos populoso (Maranhão); três apresentam maior concentração de enfermeiros nas capitais (Maranhão, Sergipe e Ceará); predomina a graduação na esfera privada com destaque para o Maranhão $(55,40 \%)$ que, ao mesmo tempo, evidencia a segunda menor oferta em cursos de pós-graduação.

As regiões Sul e Sudeste apresentam cenários similares quanto ao número de enfermeiros $\mathrm{e}$ IES de caráter privado na oferta dos cursos. $\mathrm{Na}$ mesma linha, mantém-se a superioridade das IES privadas, no entanto permanecem restritas as ofertas dos cursos de pós-graduação ao considerar a quantidade de cursos ofertados e sua relação com o número de enfermeiros registrados.

No tocante a região Centro-Oeste, à exceção do Distrito Federal, os demais estados equiparam-se quanto ao número de enfermeiros/habitantes e reiteram a concentração dessa categoria nas capitais. A despeito das IES, confirma-se a predominância das instituições de cunho privado e destaca-se o estado de Goiás com 70,0\% de escolas nessa modalidade. Por outro lado, perdura a pouca oferta de cursos de pós-graduação.

De forma geral, os dados de profissionais que concluíram o curso superior de enfermagem, de acordo com a PPEB, mostram que as políticas públicas não têm sido efetivas para desconcentrar o aparelho formador do profissional de enfermagem da região Sudeste. Esta concentração perpassa o nível superior, especialização e/ou técnico. A formação dessa força de trabalho, indispensável ao Sistema Único de Saúde, não tem sido financiada pelo setor público ${ }^{38}$.

Grande contingente da equipe de enfermagem - auxiliares, técnicos, enfermeiros graduados e especialistas, não foram, no decorrer de suas formações, contemplados pela esfera pública. Considerando a pós-graduação como um todo, a maioria dos profissionais tem formação eminentemente oriunda de IES privadas $(52,6 \%)$. No nível de lato sensu esse fenômeno é mais intenso, com $66,8 \%$ dos enfermeiros especialistas titulados no setor privado. Apenas no caso das Residências, enfermagem e/ou multiprofissional, a esfera pública se sobrepõe respondendo pela formação de $76,1 \%$ dos enfermeiros ${ }^{39}$.

Os investimentos da iniciativa privada não continua na pós-graduação stricto sensu, nível em que a demanda é menor $(19,6 \%)$ entre os enfermeiros que cursaram Mestrado Profissional, Acadêmico, Doutorado ou Pós-doutorado. Pode-se afirmar que nas modalidades de Pós-Graduação tradicionais às políticas públicas de formação são importantes estruturadoras da oferta de Mestrado Acadêmico $(87,1 \%)$ Doutorado $(93,2 \%)$ e Pós-Doutorado $(81,8 \%)$.

Outro desafio posto à formação do enfermeiro é a assimetria na localização dessas IES que comprometem a fixação e atuação do profissional nos locais onde auferem seus diplomas. Estabelece-se assim, os fluxos migratórios intra e interpaíses e, o Brasil encontra-se inserido nesse contexto.

Reitera-se que é indispensável à formação do enfermeiro a apropriação de temas macropolíticos, econômicos e sociais para lidar com demandas em saúde das fronteiras do Brasil que envolvem 569 municípios em 15.719 Km, com os países do Mercado Comum do Sul (MERCOSUL) no enfrentamento de condições adversas de saúde ${ }^{35}$, a exemplo de doenças imunopreveníveis.

Devido a relevância do enfermeiro no setor saúde, a redução desse profissional em países desenvolvidos e em desenvolvimento preocupa os 
dirigentes dos sistemas de saúde. Nesse sentido, incentivos para os enfermeiros migrarem para outros países tem agravado as lacunas dos países de origem. Problemas como insatisfação no trabalho, fragilidades ou restrição de políticas sociais, práticas disruptivas que comprometem o bem-estar social, busca por segurança e melhorias na qualidade de vida são alguns fatores que aceleram os fluxos migratórios ${ }^{40}$.

Dados do The World Health Report 2006 Working Together for Health ${ }^{38}$ evidenciam que o percentual de enfermeiros com formação estrangeira que trabalham na Austrália, Canadá, Reino Unido e Estados Unidos representa de 5 a 10\% da força de trabalho de enfermagem desses países. Em Nova Zelândia, 21\% da equipe de enfermagem são do exterior.

Kingma $^{37}$ acrescenta que na Suíça 30\% dos enfermeiros registrados são estrangeiros; em pelo menos um hospital universitário $70 \%$ são de outros países; $84 \%$ dos ingressantes no registro irlandês de enfermagem tinham formação estrangeira; excluindo-se países da União Europeia, ainda somam 60\%. No Reino Unido 37.000 enfermeiros estrangeiros esperam para cumprir os requisitos de acreditação.

Estudo $^{39}$ mostra os destinos mais requisitados: EUA, Reino Unido, Canadá e Emirados Árabes Unidos. Enfermeiros nos EUA podem ganhar até $82,7 \%$ mais do que as indianas. No Canadá e Emirados Árabes Unidos as diferenças salariais são modestas, porém melhores em até 28 e $20 \%$, respectivamente. As enfermeiras no Reino Unido estão em desvantagem salarial quando comparadas aquelas que trabalham na Índia.

Nesta polaridade entre os fluxos migratórios, a formação do profissional, a distribuição geográfica das IES, a realidade brasileira convive com o incremento dos cursos na modalidade EAD, como também, o funcionamento de cursos noturnos na formação da enfermagem.
Pesquisa do CNS, revelou que o número de vagas autorizadas pelo Ministério da Educação (MEC) para a graduação a distância, em cursos na área de saúde, totalizava em torno de $690 \mathrm{mil}$, considerando dados de junho de 2018. No entanto, inexiste consenso sobre os rumos da EAD na formação do enfermeiro no Brasil. Ao mapearem no cenário nacional os cursos de ensino superior de enfermagem nesta modalidade, identificaram que está em expansão e viabiliza o acesso ao ensino superior (graduação e pós-graduação) a um número maior de estudantes. Nessa lógica, a Enfermagem tem crescido, prioritariamente na pós-graduação, mesmo tímida em relação a outras áreas ${ }^{41}$.

\section{Considerações finais}

O cenário de empregabilidade exige um enfermeiro capaz de liderar equipes detentoras de múltiplos saberes e práticas, visão global e interconectada com os avanços tecnológicos e culturais. Muitas instituições de ensino avançam nessa ótica, contudo, outras tantas carecem de reorientações nos construtos teóricos e pedagógicos para, em um futuro próximo, assegurar que as populações detenham melhores indicadores de saúde e se identifique satisfação e valorização bidirecional entre os profissionais enfermeiros e as sociedades plurais.

Nesse sentido, considerando as distorções concernentes à distribuição desigual do contingente de profissionais enfermeiros nos Estados brasileiros, entre capitais e interiores, parece ser fundamental que o poder público tome as rédeas desse processo, ofertando cursos de enfermagem, adequados às realidades locais e às exigências de formação globais, nos territórios vazios de enfermeiros, mormente nos interiores desse Brasil continental. 


\section{Colaboradores}

MA Frota e MCMW Wermelinger: concepção e desenho. LJES Vieira e RSM Queiroz: redação e revisão crítica. FRG Ximenes Neto e RF Amorim: aprovação da versão a ser publicada.

\section{Referências}

1. Organisation for Economic Co-operation and Development (OECD). Health at a Glance 2017: OECD Indicators [livro na Internet]. Paris: OECD Publishing; 2017. [acessado 2019 Jul 14]. Disponível em: http:// dx.doi.org/10.1787/health_glance-2017-en

2. Shava GN, Tlou FN. Distributed Leadership in Education, Contemporary Issues in Educational Leadership. African Educational Res J 2018; 6(4):279-287.

3. Pinheiro R. Bridging the Local with the Global: Building a new university on the fringes of Europe. Tertiary Education and Management 2013; 19(2):144-160.

4. Nigenda G, Magaña-Valladares L, Cooper K, Ruiz-Larios JA. Recent Developments in Public Health Nursing in the Americas. Int J Environ Res Public Health 2010; 7(3):729-750.

5. Machado MH. Notas metodológicas. Enferm Foco 2016; 7(n. esp.):06-08.

6. Machado $\mathrm{MH}$, Wermelinger M, Vieira M, Oliveira E, Lemos W, Aguiar Filho W, Lacerda WF, Santos MR, Souza Junior PB, Justino E, Barbosa C. Aspectos gerais da formação da enfermagem: o perfil da formação dos enfermeiros, técnicos e auxiliares. Enferm Foco 2016; 6(2/4):15-34.

7. Carneiro LA, Porto CC. Saúde Mental nos cursos de graduação: interfaces com as diretrizes curriculares nacionais e com a reforma psiquiátrica. Cad Bras Saude Mental 2014; 6(14):150-167.

8. Winters JRF, Prado ML, Heidemann ITSB. A formação em enfermagem orientada aos princípios do Sistema Único de Saúde: percepção dos formandos. Esc Anna Nery 2016; 20(2):248-253.

9. Brasil. Conselho Nacional de Educação. Resolução CNE/CES no 3 de 7 de novembro de 2001. Institui Diretrizes Nacionais do Curso de Graduação em Enfermagem. Diário Oficial da União; 2001.

10. Marçal M, Marconsin M, Xavier J, Silveira L, Alves $\mathrm{VH}$, Lemos A. Análise dos projetos pedagógicos de cursos de graduação em enfermagem. Rev Baiana Enferm 2014; 28(2):117-125.

11. Fernandes JD, Rebouças LC. Uma década de diretrizes curriculares nacionais para a graduação em enfermagem: avanços e desafios. Rev Bras Enferm 2013; 66(n. esp.):95-101.

12. Jofré S, Sepúlveda C. Migración en Chile: nuevo desafío para la formación de competencias culturales en estudiantes de enfermería. Rev Enfermería Actual 2017; 33:1-11.

13. Broeiro P. Papel social do pensamento crítico. Rev Port Med Geral Fam 2014; 30(8):147-148.

14. Pepin JI, Myrick F. A conversation on nursing education research with the 2018 Pat Griffin Scholar, Dr Linda Fergunson. QANE/AFI 2019; 5(1):2-14.

15. Kloh D, Lima MM de, Reibnitz KS. Ethical and social commitment in the teaching plan of nursing education. Texto Contexto - Enferm 2014; 23(2):484-491.

16. Organização Mundial da Saúde (OMS). Marco para Ação em Educação Interprofissional e Prática Colaborativa [documento na Internet]. Genebra: OMS; 2010. [acessado 2019 Jul 14]. Disponível em: http:// new.paho.org/bra/images/stories/documentos/marco_para_acao.pdf $\% 20$ 
17. Brasil. Ministério da Saúde (MS). Secretaria Nacional de Assistência à Saúde. $A B C$ do SUS: doutrinas e princípios. Brasília: MS; 1990.

18. Silva KL, Sena RR, Tavares TS, Maas LW. Expansão dos cursos de Graduação em Enfermagem e mercado de trabalho: reproduzindo desigualdades? Rev Bras Enferm 2012; 65(3): 406-413.

19. Prates AAP. Universidades vs terceirização do ensino superior: a lógica da expansão do acesso com manutenção da desigualdade: o caso brasileiro. Sociologias 2007; 17:102-123.

20. Oliveira JSA, Pires DEP, Alvarez AM, Sena RR, Medeiros SM, Andrade SR. Trends in the job market of nurses in the view of managers. Rev Bras Enferm 2018; 71(1):148-155

21. Porto AMS, Soares AB. Diferenças entre expectativas e adaptação acadêmica de universitários de diversas áreas do conhecimento. Aná Psicológica 2017; 24(33):929-939.

22. Instituto Nacional de Estudos e Pesquisas Educacionais Anísio Teixeira (INEP). Saeb 2001: novas perspectivas [documento na Internet]. Brasília: Inep; 2002. [acessado 2019 Jul 14]. Disponível em: http:// portal.inep.gov.br/documents/186968/484421/SAE$\mathrm{B}+2001+$ novas+perspectivas/cd69d523-8fc6-48f48007-7a2aa4bb99bd?version $=1.1$

23. Instituto Nacional de Estudos e Pesquisas Educacionais Anísio Teixeira (INEP). Matriz de referência Enem [documento na Internet]. Brasília: MS; 2012. [acessado 2019 Jul 14]. Disponível em: http://download. inep.gov.br/educacao_basica/enem/downloads/2012/ matriz_referencia_enem.pdf

24. Instituto Nacional de Estudos e Pesquisas Educacionais Anísio Teixeira (INEP). Sinopses estatísticas da educação superior [documento na Internet]. 2013 [acessado 2019 Jul 14]. Disponível em: http://portal. inep.gov.br/superior-censosuperior-sinopse

25. Teixeira E, Vale EG, Fernandes JD, De Sordi MRL Trajetórias e tendências dos cursos de enfermagem no Brasil. Rev Bras Enferm 2006; 59(4):479-487.

26. Haddad AE, Pierantoni CR, Ristoff D, Xavier IM, Giolo J, Silva LB. A Trajetória dos Cursos de Graduação na Saúde: 1991 a 2004. Brasília: INEP/MEC; 2006.

27. Instituto Nacional de Estudos e Pesquisas Educacionais Anísio Teixeira (INEP). Censo da Educação Superior 2014: Notas Estatísticas. Brasília: MEC; 2015.

28. Sawaengdee K, Tangcharoensathien V, Theerawit T, Thungjaroenkul $\mathrm{P}$, Tawarungruang $\mathrm{C}$, Thinkhamrop B. Thai Nurse cohortstudy: cohort profiles and key findings. BMC Nursing 2016; 15:10.

29. Ferreira-Junior AR, Fontenele MEPF, Albuquerque RAS, Gomes FMAG, Rodrigues MENG. A socialização profissional no percurso de técnico a enfermeiro. Trab Educ Saude 2018; 16(3):1321-1335.

30. Peres CRFB, Marin MJS, Tonhom SFR, Barbosa PMK. Integração ensino-serviço na formação do enfermeiro no Estado de São Paulo (Brasil). REME 2018; 22:1-8.

31. Silva KL, Sena RR, Tavares TS, Belga SMMF, Maas LWD. Enfermeiros migrantes no Brasil: características demográficas, fluxos migratórios e relação com o processo de formação. Rev Latino Am Enfermagem 2016; 24:e2686.
32. World Health Organization (WHO). Health workforce requirements for universal health coverage and the Sustainable Development Goals. Hum Resources Health Observer 2016; 17:1-35.

33. Chaves LDP, Mininel VA, Silva JAM, Alves LR, Silva MF, Camelo SHH. Supervisão de enfermagem para a integralidade do cuidado. Rev Bras Enferm 2017; 70(5):1106-1111.

34. Lima ARA, Buss E, Ruiz MCS, González JS, Heck RM. Possibilidades de formação em enfermagem rural: revisão integrativa. Acta Paul Enferm 2019; 32(1):113119.

35. Guerra K, Ventura M. Bioética, imigração e assistência à saúde: tensões e convergências sobre o direito humano à saúde no Brasil na interação regional dos países. Cad Saude Colet 2017; 25(1):123-129.

36. Valizadeh S, Hasankhani H, Shojaeimotlagh V. Nurses' Immigration: causes and problems. Int J Med Res Health Sci 2016; 5(9S):486-491.

37. Kingma M. Nurses on the move: a global overview. Health Serv Res 2007; 42(3 Pt. 2):1281-1298.

38. World Health Organization (WHO). The World Health Report 2006 - Working Together for Health. Geneva: WHO; 2006

39. George $G$, Rhodes B. Is there a financial incentive to immigrate? Examining of the health worker salary gap between India and popular destination countries. Hum Resources Health Observer 2017; 15(1):74.

40. Conselho Nacional de Saúde (CNS). $1^{a}$ Conferência Nacional de Vigilância em Saúde: Relatório nacional consolidado [documento na Internet]. 2018 [acessado 2018 Maio 10]. Disponível em: http://conselho.saude. gov.br/web_cnvs/01.pdf

41. Rojo PT, Vieira SS, Zem-Mascarenhas SH, Sandor ER, Vieira CRSP. Panorama da educação à distância em enfermagem no Brasil. Rev Esc Enferm USP 2011; 45(6):1476-1480.

Artigo apresentado em 30/04/2019

Aprovado em 20/08/2019

Versão final apresentada em 20/09/2019 
